\title{
Findings on Real-Time Location Tracking by Implanting Different Mechanisms
}

\section{A. Sai Hanuman, Kanegonda Ravi Chythanya}

\begin{abstract}
Monitoring individuals or important things has never been simpler, thanks to advances in communication technology. A tracking device is an electronic unit intended to communicate its area, either in light of a sign or at set interims.Tracking devices can permit you to screen stock, find imperiled creatures, or help salvage laborers discover you in a crisis.So Real-time location tracking consistently takes on a vital job in the life of the person. Growth in advanced technology advancements such as $2 G, 3 G$, $4 G$, LoRa, and ZigBee has gained progressive changes in realtime location tracking. Technology only works when it reaches the user's every standard. This study explored the appropriate technology to track the real-time position amidst the technologies listed above.This paper proposed a model for real-time location tracking through a device that offers the ability to track the location in case of emergency. The tracking system involves the GSM-based GPRS activated unit that continuously transmits the moving object's GPS location to a mobile device that has activated the specified software and sends the SMS in an emergency to the registered mobile number.
\end{abstract}

Keywords: LoRa, Zigbee.

\section{INTRODUCTION}

The GPS-GPRS tracking system is the system that uses GPS technology to determine the exact global position whenever the distance voyaged through the GPS receiver attached to the target from the Global Positioning Satellites. The GPRS and GPS tracking system will provide data on the global position in real-time and efficiently[1]. This system uses a module consisting of a GPS receiver and a Microcontrollerinterfaced GSM modem. Google Maps is used to map the locations as follows. The GSM modem takes the region of the GPS and sends it to the GPRS server. This reveals how such innovative developments of GPS and GSM-GPRS can be intelligently integrated for a real global issue[2]. The combination of GPS and GSM technologies was first developed through the use of SMS as a GPS transmission procedure[3]. Improving GPRS invention to GSM to relay region directions to a remote server allows remote monitoring through the use of any internet-related PC.

\section{PROPOSED TRACKING SYSTEM}

The tracking system involves the GSM-based GPRS activated unit that continuously transmits the moving objects GPS location to a mobile device that has activated the

Revised Manuscript Received on March 18, 2020.

A. Sai Hanuman, Professor and Dean (Academics), GokarajuRangaraju Institute of Engineering Technology

Kanegonda Ravi Chythanya, Assistant Professor, S R Engineering College specified software and sends the SMS in an emergency situation to the registered mobile number. For the plan and usage of the proposed framework, a combination of emerging technologies has been developed.

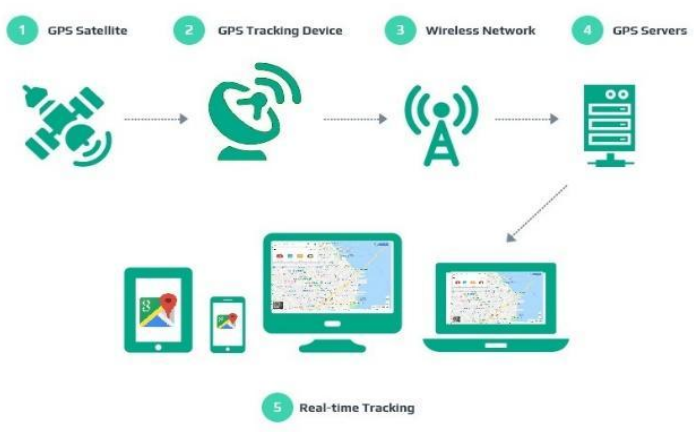

Fig 1: Design of the Tracking System

\section{Components}

The proposed tracking system provides the expected results of efficient communication and interaction between components such as the integrated SIM808 GPRS-GPS shield module with the Arduino microcontroller board and communicates via the UART serial interface.

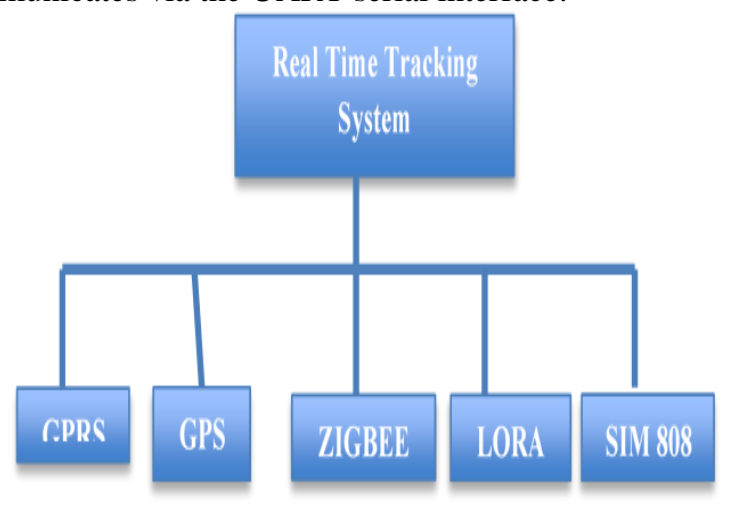

Fig 2: Components of Application

Fig 3 represents the Real-time tracking system that uses GPRS, GPS, ZIGBEE, LORA, and SIM 808 services help in to find the real-time location.

\section{LITERATURE SURVEY}

There are several tracking systems reported in literature based on GPS,GPRS, RFID, Wireless LAN, Bluetooth, LoRa etc.

SeokJu Lee, proposed a vehicle following framework utilizing GPS/GSM/GPRS innovation and a Smartphone application to offer better support and savvy answer for clients[1]. Morii et al, proposed a children Tracking System using android terminals,

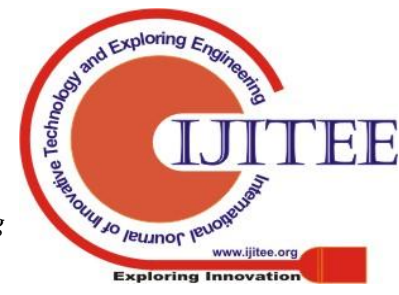




\section{Findings on Real-Time Location Tracking by Implanting Different Mechanisms}

in which Bluetooth technology was used to enable communication among android terminals and configure a Bluetooth MANET [2].

Pham Hoang Oat, proposed the improvement of vehicle following framework utilizing the Global Positioning System (GPS) and Global System for Mobile Communications (GSM) modem is embraced with the point of empowering clients to find their vehicles easily and in an advantageous way. The framework will give clients the ability to follow vehicle remotely through the versatile system[3]. J.Saranya, proposed an actualizing child following framework for each child going to class. This framework incorporates a youngster module and two beneficiary modules for getting the data about the missed kid on periodical premise [4]. M. A. Al Rashed proposed a GPS based tracking system which keeps track of the location of a vehicle and its speed based on a mobile phone text messaging system. The system is able to provide realtime text alerts for speed and location [5]. Hind Abdalsalam Abdallah Dafallah proposed a system that effectively got GPS flags, prepared and transmitted the information to the following place. In the following community the directions have been shown appropriately on Google maps, which revive naturally like clockwork to get the new area [6]. A. Omanakuttan proposed design of unique engine locking system to control seizing of vehicles using GSM and GPS technology based embedded system [7]. Ms.Sonali S. Kumbhar, proposes a speedy reacting system that helps ladies during inconvenience. At the point when somebody will disturb, she can simply press the catch and the area data is sent as a SMS alarm to not many pre-characterized numbers regarding scope and longitude [8]. Kunal Maurya proposed to plan an inserted framework which is utilized for following and situating of any vehicle by utilizing Global Positioning System (GPS) and Global framework for versatile correspondence (GSM).In this Device AT89C51 microcontroller is utilized for interfacing to different equipment peripherals. The present plan is an inserted application, which will consistently screen a moving Vehicle and report the status of the Vehicle on request.Benny Vejlgaard proposed the inclusion and limit of SigFox, LoRa, GPRS, and NB-IoT is analyzed utilizing a genuine site sending covering $8000 \mathrm{~km} 2$ in Northern Denmark.

\section{GPRS}

GPRS (General Packet Radio Service) is a packet switched mobile data service on the Global Mobile Communications System (GSM) of the $2 \mathrm{G}$ and $3 \mathrm{G}$ cellular network, expanded to use the Voice Facilities over the Communication Channel as opposed to GSM, which is not suitable for non-voice facilities due to its circuit switching capabilities. It provides its users with the fast and moderate Internet facilities. It agrees to use the GSM to use the Voice Services, but when the user is expected to transmit the data, the GPRS program must send it. It offers up to $171.2 \mathrm{kbps}$ of transmission rates. The GPRS packet transmission leaves the networks of GPRS-GSM, the packets are transported to the Internet. This therefore along with the new protocols, provides the modern transmitting and signalling method.

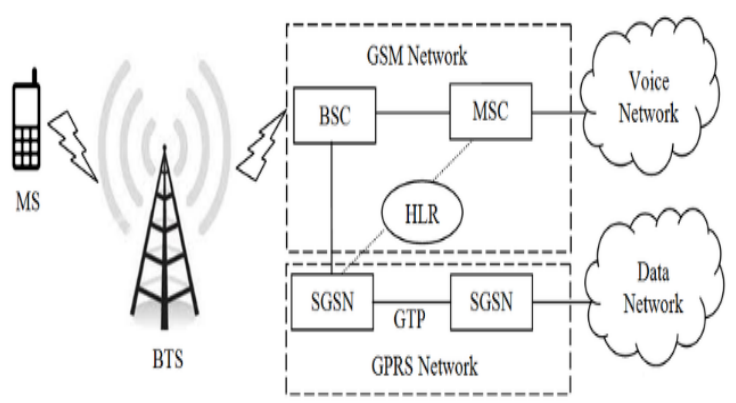

Fig 3: GPRS System

\section{Globalpositioning System}

The Global Positioning System (GPS) formerly NAVSTAR GPS is a radio navigation framework which is a space-based satellite framework which gives continuous area and time data to a beneficiary in wherever on or close to the Earth where there is an open view to at least four GPS Satellitesout of 33 satellites.

The GPS is based on the GPS satellite's known position and real-time. The GPS satellites hold the steady Atomic Clocks that are synchronized with each other as well as the ground clocks. Every hint changed in the ground is periodically adjusted. In addition, the satellite locations are also known to be excessively accurate. There are also chronometers for GPS receivers. But they're less stable and less accurate.

Each satellite uninterruptedly sends a signal which is a carrier wave comprising its position data and the current time. Meanwhile, the speed of the carrier wave is constant and not dependent on the intensity of the satellite, the time delay is equivalent to the distance between the satellite and the GPS receiver. A GPS receiver tracks four or more satellites to assess the receiver's precise location and its realtime eccentricity. At the lowest, the receiver must have four GPS satellites insight to measure four values (clock deviation from satellite time and three location coordinate values).

\section{ZIGBEE}

Zigbee is an international IEEE 802.15.4 standard designed to create high-level personal area network with tiny, lowpower radios are used to automate small-scale services that require wireless communication. Zigbee is now a Wireless Ad Hoc Network that is lower data rate, lower power, and closer proximity.

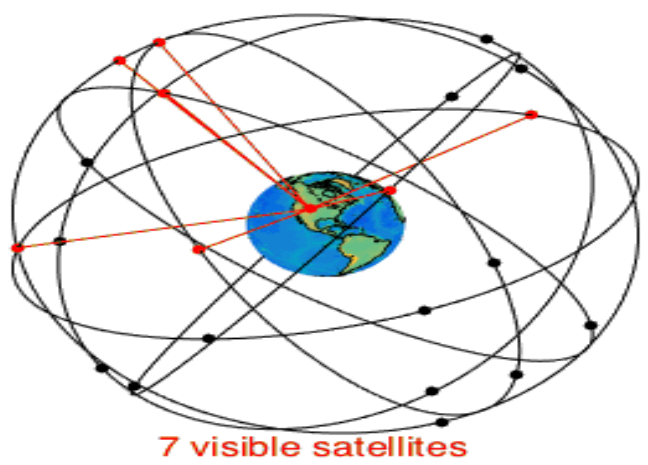

Fig 4: GPS Satellites 
Zigbee is simpler than other ad-hoc network systems such as Wi-Fi and Bluetooth and operates over long distances. Zigbee-enabled devices can communicate data over long distances by sending data between source and destination through a mesh network of Zigbee-enabled devices.

Zigbee will be built for less-speed WPANs on the media access control layer and the physical layer specified in International IEEE 802.15.4. The definition of Zigbee incorporates four additional key components: Application Layer, Network Layer, Manufacturer-defined Application Objects and ZDOs. ZDOs (Zigbee Device Objects) are responsible for certain activities, including keeping track of Zigbee device functions, requests from other users to access the networks to be handled, as well as finding the other Zigbee enabled devices and their protection.

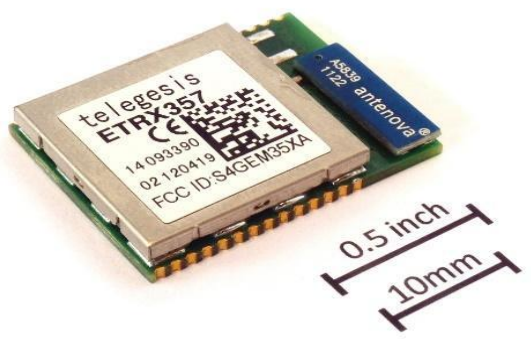

Fig 5: Zigbee Module

\section{LoRa WAN}

Long Range (LoRa) is a LPWAN (Low-Power Wide Area Network) based technology. This technology works on the modulation techniques of a CSS (Chirp Spread Spectrum) derived from Spread Spectrum. LoRa enables broadcasting of long-range data (up to $10 \mathrm{~km}$ in rural areas) with less electricity.

There are two types of LoRa: LoRa (Physical Layer) and LoRa WAN (Upper Layer).

LoRa is a trademarked, so there is no publicly available documentation, although Semtech has provided a description of the LoRa modulation and other technical features that are relevant. Like LoRa, LoRa WAN includes the upper layer requirements. LoRa WAN is a cloud basedMAC layer protocol. LoRa WAN provides the definition for the System architecture and Communication Protocol for the network. LoRa WAN is also responsible for maintaining the frequencies for the communication, data transmission rate, and power for all the LoRa devices.

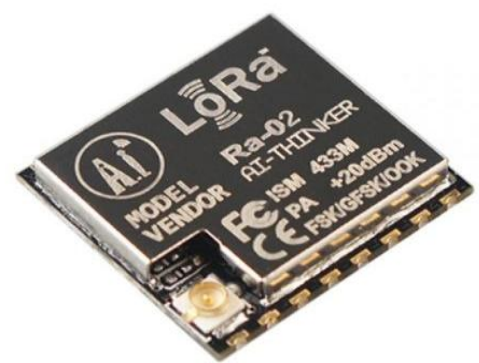

Fig 6:LoRa Module

LoRa is a trademarked, so there is no publicly available documentation, although Semtech has provided a description of the LoRa modulation and other technical features that are relevant. Like LoRa, LoRa WAN includes the upper layer requirements. LoRa WAN is a cloud based MAC layer protocol. LoRa WAN provides the definition for the System architecture and Communication Protocol for the network. LoRa WAN is also responsible for maintaining the frequencies for the communication, data transmission rate, and power for all the LoRa devices.

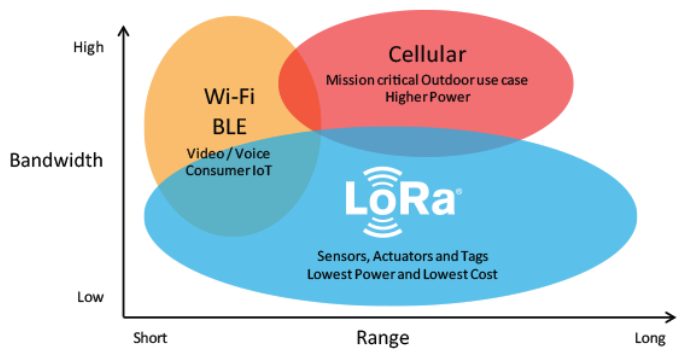

Fig 7: Comparison of the LoRa with Wifi, GPRS

\section{SIM 808 MODULE}

SIM808 is a module for GSM / GPRS 850/900/1800/1900 MHz. This module assimilates GPS to the device's navigation. This package contains a SIM toolkit that allows easy implantation of the SIM card. A GPRS / GSM modem with SIM808 module allows the development of data connections via a standard USB interface over the GSM network. SIM808 is based on the power-saving technique; thus, the power consumption in the sleep mode is very low as $1.0 \mathrm{~mA}$.

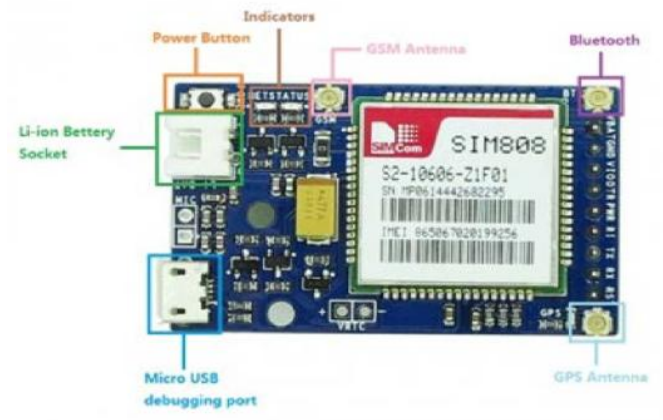

IV. ANALYSIS AND COMPARISON

\begin{tabular}{|c|c|c|c|c|}
\hline Parameter & GSM & GPRS & ZigBee & LoRaWAN \\
\hline Data rates & 14.4 kbps & $57.6 \mathrm{kbps}$ & \begin{tabular}{|l|l|}
20,40, & 250 \\
kbps & \\
\end{tabular} & $0.3-22 \mathrm{kbps}$ \\
\hline Carrier Size & $200 \mathrm{kHz}$ & $200 \mathrm{kHz}$ & $\begin{array}{l}868 \mathrm{MHz}, 915 \\
\mathrm{MHz}, 2.4 \mathrm{GHz}\end{array}$ & $\begin{array}{l}\text { 863-870 } \mathrm{MHz}, 902-928 \\
\mathrm{MHz}, 779-787 \mathrm{MHz}\end{array}$ \\
\hline Range & $35 \mathrm{KMS}$ & $25 \mathrm{KM}$ & 10-100 mts & $\begin{array}{l}2.5 \mathrm{Kms} \text { (Urban), } 15 \mathrm{Km} \\
\text { (suburbant) }\end{array}$ \\
\hline Power Consumption & $\begin{array}{l}1 \mu \mathrm{A} \text { to } 480 \\
\mathrm{~mA}\end{array}$ & $2347 \mathrm{~mA}$ & $50 \mathrm{~mA}$ & $12 \mathrm{~mA}$ \\
\hline Features & SMS & MMS & NA & NA \\
\hline
\end{tabular}

\section{Published By:}




\section{Findings on Real-Time Location Tracking by Implanting Different Mechanisms}

Findings are tabulated as above by evaluating various technologies such as GSM, GPRS, ZigBee and LoRaWAN.

Thus, monitoring is done by GSM, data transmission at a rate of $14.4 \mathrm{kbps}$ and carrier size is $200 \mathrm{kHz}$, covering a range of $35 \mathrm{kms}$ with power consumption between $1 \mathrm{uA}$ and $480 \mathrm{~mA}$. The location information can be transmitted by SMS.

In the case of GPRS software, a carrier size of $200 \mathrm{kHz}$ can be transmitted at a rate of $57.6 \mathrm{kbps}$. This technology will cover the $25 \mathrm{kms}$ range, but it raises the level of consumption to $2347 \mathrm{~mA}$. Via MMS, we can share location details.

ZigBee transmits data at $20 \mathrm{kbps}, 40 \mathrm{kbps}, 25 \mathrm{kbps}$ speeds and can be transmitted at $868 \mathrm{MHz}, 915 \mathrm{MHz}, 2.4 \mathrm{GHz}$ carrier frequency. This can cover the power consumption spectrum up to $10-100 \mathrm{mts}$ with $5 \mathrm{~mA}$.

LoRaWAN transfers data with carrier size of $863-870 \mathrm{MHz}$, $902-928 \mathrm{MHz}, 779-787 \mathrm{MHz}$ at a rate of $0.3-22 \mathrm{kbps}$. This technology will cover up to $2-5 \mathrm{kms}$ in urban areas and $15 \mathrm{kms}$ in suburban areas with the power consumption of $12 \mathrm{~mA}$.

\section{CONCLUSION}

We also examined various types of technology in this survey that are used to monitor and communicate the position in real-time. We considered the LoRaWan module to be very useful for our work because more distance can be achieved.

\section{ACKNOWLEDGEMENTS}

This research was supported by TEQIP-III, JNTU Hyderabad under Collaborative Research Scheme. We thank the team of TEQIP-III for their generous support for this research.

We would like to thank Mr. Balaji Sir and Radha Mohan Sir of Electronics and Communication Engineering for extending their support.

We would also like to show our gratitude to our management to provide us with the required environment to perform this research.

\section{REFERENCES}

1. S. Ahmed et al, "Real-Time Vehicle Tracking System," M.S. thesis, Dept. Elect. and Electron. Eng., BRAC Univ., Dhaka, Bangladesh, 2015.

2. A. Ajagbe et al, "The Use of Global System of Mobile Communication (GSM) Among University Students in Malaysia," International Journal of Innovation, Management and Technology (IJIMT), Vol. 2, No. 6, pp. 512-518, Dec. 2011.

3. A. El-Rabbany, "Introduction to GPS: The Global Positioning System," Boston, USA: Artech House, 2002.

4. "GPRS General Packet Radio Service," Usha Communication Technology Ltd., London, UK, June 2000.

5. "SIM808 Hardware Design V1.00," Shanghai SIMCom Wireless Solution Ltd., Version No. 1.00, Shanghai, China, Mar. 27, 2014.

6. L. Fried, "Adafruit FONA 808 Cellular + GPS Breakout," Adafruit Industries Co., New York, USA, Feb. 2016.

7. ALHMIEDAT, T.A. and YANG, S.H., 2008, "A ZigBee-based mobile tracking system through wireless sensor networks", International Journal of Advanced Mechatronic Systems, 1 (1), pp.6370 .

8. Saima Safdar, Anwar Zeb, Ajmal Khan and Zeeshan Kaleem, "Android Based Vehicle Tracking System", EAI Endorsed Transactions on Energy Web and Information Technologies.

9. Mr. Prasad Rajendra Joshi, Mr. Vishal Vasudeo Patil, Mr. Piyush Sanjay Koli, "Device Tracking using Embedded GPS and Zigbee Technology", International Journal for Technological Research in
Engineering, Volume 4, Issue 8, April-2017, ISSN (Online): 2347 4718

10. https://en.wikipedia.org/wiki/Global_Positioning_System

11. K.-T. Song and C.-C. Yang, "Front Vehicle Tracking Using Scene Analysis," in Proceedings of the IEEE International Conference on Mechatronics \& Automation, 2005.

12. A. Alexe and R.Ezhilarasie, "Cloud Computing Based Vehicle Tracking Information Systems," IJCST, vol. 2, no. 1, March 2011.

13. W. El-Medany, A. Al-Omary, R. Al-Hakim, S. Al-Irhayim and M. Nusaif, "A Cost-Effective Real-Time Tracking System Prototype Using Integrated GPS/GPRS Module," in 6th International Conference on In Wireless and Mobile Communications (ICWMC), 2010.

14. H. Lee, D. Kim, D. Kim and S. Y. Bang, "Real-time automatic vehicle management system using vehicle tracking and car plate number identification," in International Conference on Multimedia and Expo. ICME '03, 2003.

15. "The Microprocessors," Connect in, 08 February 2005. [Online] Available:

http://connect.in.com/microprocessor/photosmicroprocessor916bf2805ceb0ac0.html. [Accessed 1310 2013].

16. K. Aravind, T. Chakravarty, M. Chandra and P. Balamuralidhar, "On the architecture of vehicle tracking system using wireless sensor devices," in International Conference on Ultra-Modern Telecommunications\&Workshops ICUMT, St. Petersburg, October 2009.

17. I. Almomani, N. Alkhalil, E. Ahmad and R. Jodeh, "Ubiquitous GPS vehicle tracking and management system," in IEEE Jordan Conference on Applied Electrical Engineering and Computing Technologies (AEECT), Amman, December 2011.

18. M. Honda, M. Murata and Y. Mizukura, "GPS Precise Point Positioning Methods Using IGS Products for Vehicular Navigation Application," in International Joint Conference SICE-ICASE, Busan, October 2006.

19. P. Misra and P. Enge, "Global Positioning System: Signals, Measurements, and Performance," in Massachusetts, Ganga-Jamuna Press, 2001.

20. Miss. Pratibha, L. Yadav, Prof. Sanjay S. Badhe, Prof. Santosh G Bari, 2016, 'Study and Literature Survey for Safety Applications: Intelligent Transport System (ITS)', 5940 International Journal of Advanced Research in Computer and Communication Engineering Vol. 5

21. Newman-Askins, Raechelle and Ferreira, Luis and Bunker, Jonathan M, 'Intelligent transport systems evaluation: From theory to practice', Proceedings 21st ARRB and 11th REAAA Conference.

22. Shanzhi Chen, Hui Xu, Dake Liu, Bo Hu, and Hucheng Wang, 2014 'A Vision of IoT: Applications, Challenges and Opportunities With China Perspective', Internet Of Things Journal, Vol.1,No. 4.

23. SeokJu Lee, GirmaTewolde, Jaerock Kwon "Design and Implementation of Vehicle Tracking System Using GPS/GSM/GPRS Technology and Smartphone Application", Electrical and Computer Engineering, Kettering University, IEEE, USA,2014.

24. Mori,Y.,Kojima,H.,Kohno, E.,Inoue.,Ohta,T.,Kakuda,Y.,Ito,A.,”A Self-Configurable New Generation Children Tracking System Based on Mobile Ad Hoc Networks consisting of Android Mobile Terminals",10th International Symposium on autonomous Decentralized Systems (ISADS),pp.339-342,23-27 March,Japan,2011.

25. Pham Hoang Oat, MichealDrieberg and Nguyen Chi Cuong "Development of Vehicle Tracking System using GPS and GSM Modem" UniversitiTeknologi PETRONAS, Malaysia IEEE,2013.

26. J.Saranya , J.Selvakumar" Implementation of Children Tracking System on Android Mobile Terminals" International conference on Communication and Signal Processing, April 3-5, 2013, India.

27. M. A. Al Rashed, Ousmane Abdoulaye Oumar, Damanjit Singh "A real time GSM/GPS based tracking system based on GSM mobile phone" IEEE,2013

28. Hind Abdalsalam Abdallah Dafallah Design and implementation of an accurate real time GPS tracking system", IEEE,2014

29. A. Omanakuttan1, D. Sreedhar2, A. Manoj3, A. Achankunju4, CM. Cherian" GPS and GSM Based Engine Locking System Using Smart Password"IJCSE,2017. 
30. Ms.Sonali S. KumbharMs.Sonal K.Jadhav2, Ms. Prajakta A.Nalawade3 ,Ms. Tamanna Y.Mutawalli4” Women Security System Using GSM And GPS” IRJET ,Mar 2018.

31. Kunal Maurya 1, Mandeep Singh 2, Neelu Jain” Real Time Vehicle Tracking System using GSM and GPS Technology- An Anti-theft Tracking System" IJECSE, Volume1,Number 3Kunal Maurya et al.

32. Benny Vejlgaard1, Mads Lauridsen1, Huan Nguyen1, Istv'an Z. Kov'acs2, Preben Mogensen12, Mads Sørensen" Coverage and Capacity Analysis of Sigfox, LoRa, GPRS, and NB-IoT",IEEE,2017.

\section{AUTHORS PROFILE}

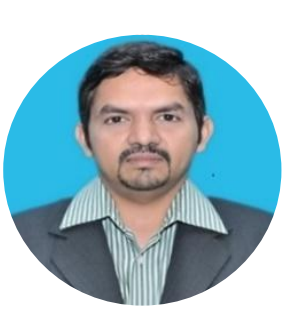

Dr. Akundi Sai Hanuman, Professor of Computer Science and Engineering, completed his Ph.D. from Acharya Nagarjuna University, Guntur in 2012. He has over 22 years of experience in Academic, Industry and Research. Dr.Akundi Sai Hanuman's Research interests include Data Clustering, Data Sciences, Machine Learning, Optimization Techniques and Distributed Systems. Currently Dr. Sai Hanuman is acting as Dean Academics, GRIET since December 2018. Previously he worked as Dean of Examinations, Head of the Department, Chairman BOS and Convener for National Level cultural festival PULSE 2013.

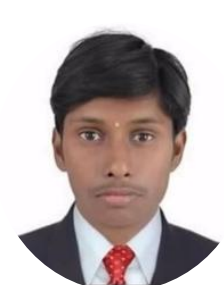

Mr. Kanegonda Ravi Chythanya, Assistant Professor of Computer Science and Engineering, pursuing his Ph.D. from JNTUH, Hyderabad. He has over 9+ years of experience in Academic and Research. Mr. Kanegonda Ravi Chythanya's Research interests include Computer Networks Bog Data Analytics, Artificial Intelligence, Machine Learning and Wireless Sensor Networks. Currently Mr. Kanegonda Ravi Chythanya is working as Assistant Professor in Computer Science and Engineering, S R Engineering College, Warangal. Previously he worked as a co-coordinator for a DST sponsored Two Week FDP on Blockchain Technologies and coordinator for a DST-NIMAT sponsored Six Week Technology Entrepreneurship Development Program on Internet of Things. 